\title{
VOLTAMETRIA DE VARREDURA LINEAR ANÓDICA EM EFLUENTES CONTAMINADOS COM SULFETO DE HIDROGÊNIO $\left(\mathrm{H}_{2} \mathrm{~S}\right)$
}

\author{
Anodic Linear Scan Voltametry in Effluents Contaminated with Hydrogen Sulfide \\ $\left(\mathrm{H}_{2} \mathrm{~S}\right)$
}

Voltamperometría de Barrido Lineal Anódica en Efluentes Contaminados con Sulfuro de Hidrógeno $\left(\mathrm{H}_{2} \mathrm{~S}\right)$

\section{Ana Flavia Ariello*1, Renata Bachmann Guimarães Valt ${ }^{2}$, Marcelo Kaminski Lenzi ${ }^{3}$, Haroldo de Araújo Ponte ${ }^{4}$}

${ }^{1}$ Programa de Pós-Graduação de Engenharia e Ciência de Materiais (PIPE), Universidade Federal do Paraná, Curitiba - PR, Brasil.

${ }^{2}$ Programa de Pós-Graduação de Engenharia Mecânica (PGMEC), PRH 12.1/PRH-ANP, Universidade Federal do Paraná, Curitiba - PR, Brasil.

${ }^{3}$ Departamento de Engenharia Química, Laboratório de Engenharia de Sistemas Fracionários (LESF), Universidade Federal do Paraná, Curitiba - PR, Brasil

${ }^{4}$ Departamento de Engenharia Química, Laboratório de Tecnologia Ambiental (LTA), Universidade Federal do Paraná, Curitiba - PR, Brasil.

*Correspondência: Universidade Federal do Paraná, Campus Centro Politécnico, Av. Cel. Francisco H. dos Santos, Jardim das Américas, Curitiba, Paraná, Brasil.CEP:81.531-990.e-mail flavia_ariello@hotmail.com.

\section{Artigo recebido em 04/03/2021 aprovado em 10/06/2021 publicado em 28/06/2021.}

\section{RESUMO}

Diante da característica altamente tóxica apresentada pelo sulfeto de hidrogênio, subproduto de diversos processos industriais, a sua remoção de um efluente torna-se indispensável. Assim sendo, avaliou-se o efeito eletroquímico da oxidação de $\mathrm{H}_{2} \mathrm{~S}$, buscando-se obter a faixa de potencial e o potencial de pico em que ocorrem as reações de oxidação do contaminante. Para tanto, utilizou-se a técnica de voltametria de varredura linear anódica com o emprego de eletrodo de trabalho rotatório de carbono vítreo reticulado. Utilizando-se de uma solução sintética em três concentrações, foram variadas as velocidades de varredura para rotações distintas do eletrodo de trabalho entre os potenciais de $-0,5 \mathrm{~V}$ (vs. ECS) e 1,0 V (vs. ECS), por meio de um potenciotato/galvanostato acoplado à um computador. Os resultados obtidos indicam que os potenciais, nos quais ocorrem as reações de interesse, ficam próximos a $0,3 \mathrm{~V}$ (vs. ECS), o que sugere a presença das mesmas reações para todos os casos analisados. Observouse nos voltamogramas um aumento proporcional da corrente de pico com a taxa de varredura e concentração, indicando o controle do sistema por transporte de massa. Assim, o presente estudo revela que o enxofre elementar não passiva a superfície do eletrodo e que há a possibilidade de utilização de eletrodos de carbono vítreo reticulado para oxidar eletroquimicamente íons sulfeto em soluções aquosas.

Palavras-chave: Eletrodo de Carbono, Sulfeto de Hidrogênio, Voltametria de Varredura Linear.

\section{ABSTRACT}

In view of the highly toxic characteristic presented by hydrogen sulfide, a by-product of several industrial processes, its removal from an effluent becomes indispensable. Therefore, the electrochemical effect of $\mathrm{H}_{2} \mathrm{~S}$ oxidation was evaluated, seeking to obtain the potential range and peak potential at which the oxidation reactions of the contaminant occur. For that, we used the anodic linear sweep voltammetry technique with 
the use of a reticulated vitreous carbon rotary working electrode. Using a synthetic solution in three concentrations, the scanning speeds for different rotations of the working electrode were varied between the potentials of $-0.5 \mathrm{~V}$ (vs. ECS) and $1.0 \mathrm{~V}$ (vs. ECS), for using a potentiotate/galvanostat coupled to a computer. The results obtained indicate that the potentials, in which the reactions of interest occur, are close to $0.3 \mathrm{~V}$ (vs. ECS), which suggests the presence of the same reactions for all analyzed cases. It was observed in the voltamograms a proportional increase of the peak current with the sweep rate and concentration, indicating the control of the system by mass transport. Thus, the present study reveals that elemental sulfur does not passivate the electrode surface and that there is the possibility of using crosslinked glassy carbon electrodes to electrochemically oxidize sulfide ions in aqueous solutions.

Keywords: Carbon Electrode, Hydrogen Sulfide, Linear Sweep Voltammetry.

\section{RESUMEN}

Dada la característica altamente tóxica que presenta el sulfuro de hidrógeno, un subproducto de varios procesos industriales, su eliminación de un efluente se vuelve indispensable. Por lo tanto, se evaluó el efecto electroquímico de la oxidación del $\mathrm{H}_{2} \mathrm{~S}$, buscando obtener el rango de potencial y el pico de potencial en el que ocurren las reacciones de oxidación del contaminante. Para eso, utilizamos la técnica de voltamperometría de barrido lineal anódico con el uso de un electrodo de trabajo rotativo de carbono vítreo reticulado. Usando una solución sintética en tres concentraciones, las velocidades de escaneo para diferentes rotaciones del electrodo de trabajo se variaron entre los potenciales de - $0.5 \mathrm{~V}$ (frente a ECS) y $1.0 \mathrm{~V}$ (frente a ECS), para usar un potenciómetro/galvanostato acoplado a un ordenador. Los resultados obtenidos indican que los potenciales, en los que ocurren las reacciones de interés, son cercanos a $0.3 \mathrm{~V}$ (frente a ECS), lo que sugiere la presencia de las mismas reacciones para todos los casos analizados. Se observó en los voltamogramas un aumento proporcional del pico de corriente con la tasa de barrido y concentración, indicando el control del sistema por transporte de masa. Así, el presente estudio revela que el azufre elemental no pasiva la superficie del electrodo y que existe la posibilidad de utilizar electrodos de carbono vítreo reticulado para oxidar electroquímicamente iones sulfuro en soluciones acuosas.

Descriptores: Electrodo de Carbono, Sulfuro de Hidrógeno, Voltamperometría de Barrido Lineal.

\section{INTRODUÇÃO}

Sulfeto de hidrogênio $\left(\mathrm{H}_{2} \mathrm{~S}\right)$ é um subproduto contaminante originado de diversos processos industriais como em refino de petróleo, em indústrias de papel e celulose, em curtumes, em abatedouros, em fertilizantes e até mesmo em estações de tratamento de esgoto (BERNÉ e CORDONNIER, 1995; ATEYA e AL-KHARAFI, 2002; PIKAAR, 2011; LAM et al., 2012; MATTIUSI et al., 2015; FORNÉS e BISANG, 2017). Ou seja, um número significativo de efluentes de processos industriais envolve a contaminação por $\mathrm{H}_{2} \mathrm{~S}$, causando danos à saúde, ao meio ambiente, e comprometimento da integridade de equipamentos industriais (JÜTTNER et al., 2000; DUTTA et al., 2010; MATTIUSI et al., 2015; FORNÉS e BISANG, 2017; PERRY et al., 2020).
$\mathrm{Na}$ forma aquosa o $\mathrm{H}_{2} \mathrm{~S}$ é um diácido fraco e dissocia-se em água a $25^{\circ} \mathrm{C}$, formando as espécies químicas como íon bissulfeto $\left(\mathrm{HS}^{-}\right)$e íon sulfeto $\left(\mathrm{S}^{-2}\right)$, sendo o $\mathrm{HS}^{-}$a espécie efetivamente corrosiva do sistema. A corrosão associada ao sulfeto de hidrogênio pode causar a deterioração total, parcial, superficial ou estrutural de materiais por ataque químico $\left(\mathrm{H}_{2} \mathrm{~S}\right.$ gasoso diretamente sobre o material metálico) ou eletroquímico (PIKAAR et al., 2015; MAINIER et al., 2007).

Técnicas físico-químicas convencionais como oxidação química (com agentes como oxigênio, cloro, hipoclorito, ozônio, peróxido de hidrogênio) ou conversão catalítica (como processo Claus) são utilizadas para redução de $\mathrm{H}_{2} \mathrm{~S}$, entretanto apresentam desvantagens como uso de altas quantidades de 
reagentes químicos e grande demanda de energia (PIKAAR et al., 2015).

Assim, o estudo de estratégias alternativas de remoção de $\mathrm{H}_{2} \mathrm{~S}$, como oxidação eletroquímica, se apresentam benéficas pois não necessitam da adição de reagentes de custo elevado e não geram poluentes secundários (JÜTTER et al., 2000).

Ânodos constituídos em base de carbono vêm sendo materiais promissores para oxidação de $\mathrm{H}_{2} \mathrm{~S}$. Isso se deve ao baixo custo e possibilidade de oxidar seletivamente sulfeto em enxofre elementar a baixos potenciais anódicos (ATEYA e AL-KHARAFI, 2002; PIKAAR et al., 2015). O eletrodo de carbono vítreo reticulado (CVR) apresenta alta condutividade térmica e elétrica. Entre outras características deste material destacam-se, a alta resistência à corrosão, as altas porosidades e consequentemente altas áreas superficiais. O CVR é amplamente utilizado, em altas densidades de corrente e células eletroquímicas de baixo volume, onde existe a necessidade de manter os poros preenchidos de eletrólito (WALSH et al., 2016; PERRY et al., 2020).

Os resultados de estudos de oxidação eletroquímica de $\mathrm{H}_{2} \mathrm{~S}$, em células de pequenos volumes, associado à técnica eletroquímica de voltametria utilizando eletrodos a base de carbono podem ser úteis para um futuro aumento de capacidade das células (MATTIUSI et al., 2015). A partir desses resultados é possível ampliar a análise do comportamento da oxidação de $\mathrm{H}_{2} \mathrm{~S}$ em compartimentos de volumes maiores.

O objetivo deste trabalho é avaliar a ocorrência da conversão eletroquímica presente em solução sintética e a possível passivação do eletrodo por enxofre elementar. Além disso, identificar a cinética eletroquímica do processo, se o controle ocorre por transporte de carga ou por transporte de massa. A importância deste estudo se dá pela possibilidade de emprego desta técnica para o tratamento desde esgotos a efluentes industriais, cujas concentrações do referido poluente podem variar de baixos a altos valores, respectivamente.

\section{MATERIAIS E MÉTODOS}

Preparação do eletrólito/solução sintética: a solução sintética de sulfeto de hidrogênio dissolvido, ou seja, o eletrólito a ser utilizado nos experimentos, é composto de uma solução preparada a partir de sulfeto de sódio nonaidratado $\left(\mathrm{Na}_{2} \mathrm{~S} \cdot 9 \mathrm{H}_{2} \mathrm{O}\right)$ (Reagen - $99 \%$ pureza) e água destilada desoxigenada por injeção de nitrogênio $\left(\mathrm{N}_{2}\right)$ durante 30 minutos (ATEYA et al., 2005; DUTTA et al., 2008; MATTIUSI et al., 2015; NTAGIA et al., 2019). Como eletrólito suporte da solução, conforme Brett (1996), foi utilizado $\mathrm{NaCl}$ a $0,1 \mathrm{~mol} \cdot \mathrm{L}^{-1}$ (Synth - $99 \%$ pureza), temperatura de $25^{\circ} \mathrm{C}$.

Percheron et al. (1996), Dutta et al. (2010), Mattiusi et al. (2015) e Ntagia et al. (2019) utilizaram $\mathrm{Na}_{2} \mathrm{~S} \cdot 9 \mathrm{H}_{2} \mathrm{O}$ na obtenção de suas soluções. A justificativa para utilizar essa metodologia em laboratório deve-se à segurança operacional, evitando a utilização do $\mathrm{H}_{2} \mathrm{~S}$ gasoso, devido à sua alta toxicidade.

A solução sintética foi preparada e então transferida para a célula eletroquímica. Toda a transferência de solução foi realizada por diferença de pressão positiva de $\mathrm{N}_{2}$, a uma temperatura de $25^{\circ} \mathrm{C}$.

Medidas voltamétricas: A voltametria é uma técnica eletroquímica que registra as informações, qualitativas e quantitativas, de corrente em função do potencial de uma dada espécie química. As curvas denominadas voltamogramas são obtidas durante a eletrólise da espécie em uma célula eletroquímica composta de 3 eletrodos. O potencial é aplicado variando uma velocidade em função do tempo, ou seja, em forma de varredura (BRETT, 1996; MATTIUSI, 2012) 
Para as três concentrações a serem analisadas, de $30 \mathrm{mg} \cdot \mathrm{L}^{-1}, 300 \mathrm{mg} \cdot \mathrm{L}^{-1}$ e $3000 \mathrm{mg} \cdot \mathrm{L}^{-1}$, foram utilizadas três velocidades de varredura: $1 \mathrm{mV} \cdot \mathrm{s}^{-1}$, $5 \mathrm{mV} \cdot \mathrm{s}^{-1}$ e $10 \mathrm{mV} \cdot \mathrm{s}^{-1} \mathrm{e}$, para cada velocidade, três rotações distintas do eletrodo de trabalho: sem rotação, $50 \mathrm{rpm}$ e $100 \mathrm{rpm}$. A varredura anódica foi realizada, entre os potenciais -0,5 V (vs. ECS) e 1,0 V (vs. ECS), por meio do galvanostato/potenciostato Gamry (ZRA Reference 600) acoplado à um computador para registro dos voltamogramas pelo software Gamry Instrument Manager.

Aparato experimental: A Fig. 1 apresenta o aparato experimental utilizado nos ensaios. Os componentes do sistema utilizados foram:

- Célula convencional eletroquímica de 100 mL com 3 orifícios: para o eletrodo disco rotatório (eletrodo de trabalho) (onde ocorrem as reações de oxidação), para o eletrodo calomelano saturado (ECS) (eletrodo de referência) (para medidas relativas de potencial) e para o eletrodo de platina (contra-eletrodo);

- Eletrodo de trabalho disco rotatório de carbono vítreo reticulado de área superficial de $0,126 \mathrm{~cm}^{2}$;

- Eletrodo de referência tipo calomelano (ECS) $\left(\mathrm{Hg} / \mathrm{Hg}_{2} \mathrm{Cl}_{2}\right), \quad \mathrm{KCl}$ saturado, marca: Analyser (modelo 3A41-FH), potencial de $+0,241 \mathrm{~V}$ em relação ao eletrodo padrão de hidrogênio;

- Eletrodo auxiliar de platina em espiral;

- Potenciostato Gamry (ZRA Reference 600). Software para aquisição de dados: Gamry Instrument Manager.

Análise química e quantificação: A quantificação dos íons foi realizada pelo método analítico de cromatografia iônica (Metrohm - modelo: 882 Compact IC), empregando como eluente a solução contendo: carbonato de sódio $\left(\mathrm{Na}_{2} \mathrm{CO}_{3}\right)$ a $3,2 \mathrm{mmol} \cdot \mathrm{L}$ ${ }^{1}$ (Synth - pureza: $\geq 98 \%$ ) e bicarbonato de sódio $\left(\mathrm{NaHCO}_{3}\right)$ a $1 \mathrm{mmol} \cdot \mathrm{L}^{-1}($ Synth - pureza: $\geq 99 \%)$. No sistema de supressão da coluna (Metrohm Suppressor Module - MSM), emprega-se uma solução de $50 \mathrm{mmol} \cdot \mathrm{L}^{-1}$ de ácido sulfúrico $\left(\mathrm{H}_{2} \mathrm{SO}_{4}\right)$ (Synth pureza: $\geq 99 \%$ ).

Figura 1. Esquema do aparato experimental utilizado nos ensaios de voltametria de varredura linear.

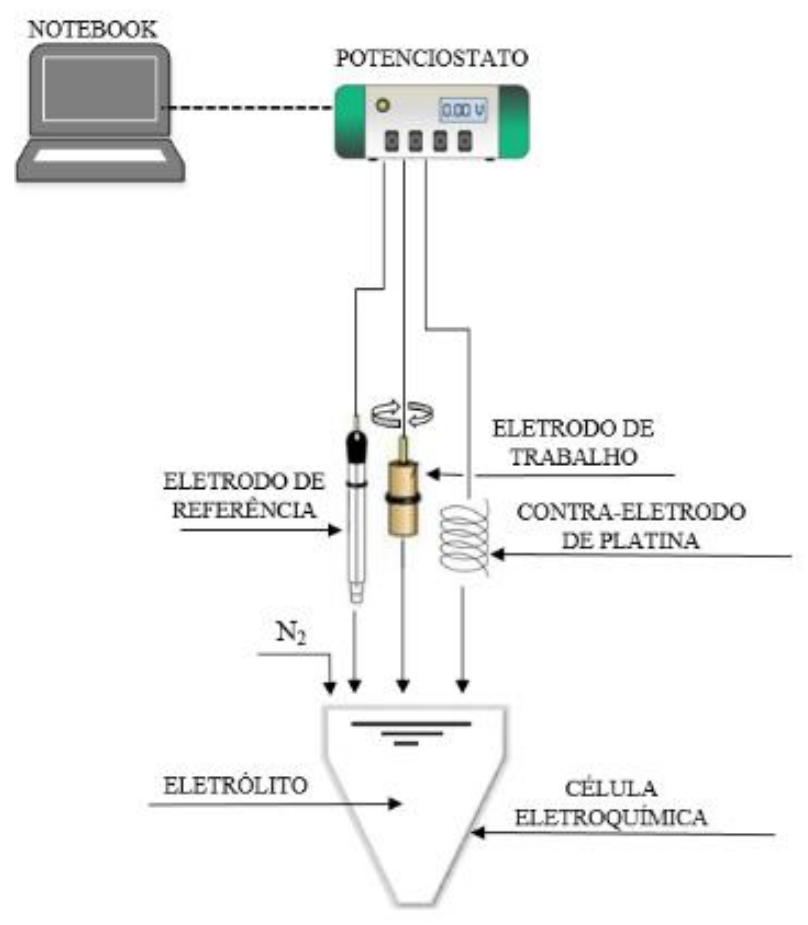

Para determinar a concentração de $\mathrm{SO}_{4}{ }^{2-} \mathrm{e}$ $\mathrm{S}_{2} \mathrm{O}_{3}{ }^{2-}$ foi realizada a diluição de $1 / 50$ da alíquota em água destilada. Em sequência, para evitar a oxidação da amostra realiza-se a análise por cromatografia iônica.

Segundo Percheron et al. (1996), Dutta et al. (2009), Ntagia et al. (2019), a quantificação do sulfeto $\left(\mathrm{S}^{2-}\right)$ não reagido deve ser realizada por oxidação com peróxido de hidrogênio $\left(\mathrm{H}_{2} \mathrm{O}_{2}\right)$ a $\mathrm{SO}_{4}{ }^{2-}$.

Assim, numa alíquota de $8 \mathrm{~mL}$ da amostra foi adicionado $0,4 \mathrm{~mL}$ de $\mathrm{NaOH}\left(6 \mathrm{~mol} \cdot \mathrm{L}^{-1}\right)(\mathrm{Neon}-$ pureza: $98 \%$ ) para estabilização ( $\mathrm{pH}>12$ ) e $2 \mathrm{~mL}$ de peróxido de hidrogênio $\left(\mathrm{H}_{2} \mathrm{O}_{2}\right)(35 \%)$ (Synth). Após 10 minutos, uma alíquota de $1 \mathrm{~mL}$ foi diluída na proporção de 1/100 em água destilada, e realizada a análise por cromatografia iônica. A diferença de $\mathrm{SO}_{4}{ }^{2-}$ após oxidação com $\mathrm{H}_{2} \mathrm{O}_{2}$ e antes da oxidação foi 
considerada como uma soma de polisulfetos, enxofre elementar e $\mathrm{S}^{2-}$ (VAIOPOULO et al., 2016).

\section{RESULTADOS E DISCUSSÃO}

O estudo voltamétrico das reações de oxidação de $\mathrm{H}_{2} \mathrm{~S}$ é fundamental para determinação dos valores de potencial a serem aplicados em compartimentos de volumes maiores, como o utilizado por Mattiusi et al. (2015). Os voltamogramas são curvas de polarização que indicam as zonas de controle de reação por transferência de massa ou por transferência de carga.

Uma reação eletroquímica ocorre em três etapas básicas. A etapa correspondente ao transporte de massa da espécie reagente que se aproxima da superfície do eletrodo. A etapa que ocorre a transferência de carga para superfície do eletrodo ocasionando a oxidação da espécie reagente. E a etapa onde a espécie reagida sofre dessorção e é transportada de volta ao seio da solução. A etapa mais lenta é a determinante da taxa de velocidade e do tipo de controle do processo, se por transporte de carga ou por transporte de massa (BARD e FAULKNER, 2001; SCOTT, 1995).

A Fig. 2 demonstra a relação das porções da curva de polarização às etapas determinantes da taxa. Observa-se nesta figura que, em baixas correntes aplicadas as reações são governadas por transferência de carga (TC), ou seja, toda carga fornecida ao sistema reage imediatamente com os íons presentes na solução, assim diz-se que o controle do processo ocorre por ativação (zona 1) (PLETCHER e WALSH, 1990; COEURET, 1992). A porção horizontal refere-se à corrente limite $\left(\mathrm{i}_{\mathrm{L}}\right)$ (ou corrente de pico), onde a reação ocorre sob controle de transporte de massa (TM), e independe do potencial (zona 3). Logo, diz-se que o controle do processo ocorre por transporte de matéria (SCOTT, 1995). A zona 2 representada na Fig. 2 corresponde ao controle misto, o qual ocorrem de forma simultâneas ambos os fenômenos anteriores (COEURET, 1992).

Assim, para obtenção das curvas de polarização, a variação de potencial aplicado foi limitada no sentido da oxidação na faixa de interesse, compreendida entre $-0,5 \mathrm{~V}$ e $1,0 \mathrm{~V}$, das reações de oxidação de $\mathrm{H}_{2} \mathrm{~S}$.

Figura 2. Curva de polarização e zonas de controle (adaptado de Coeuret, 1992).

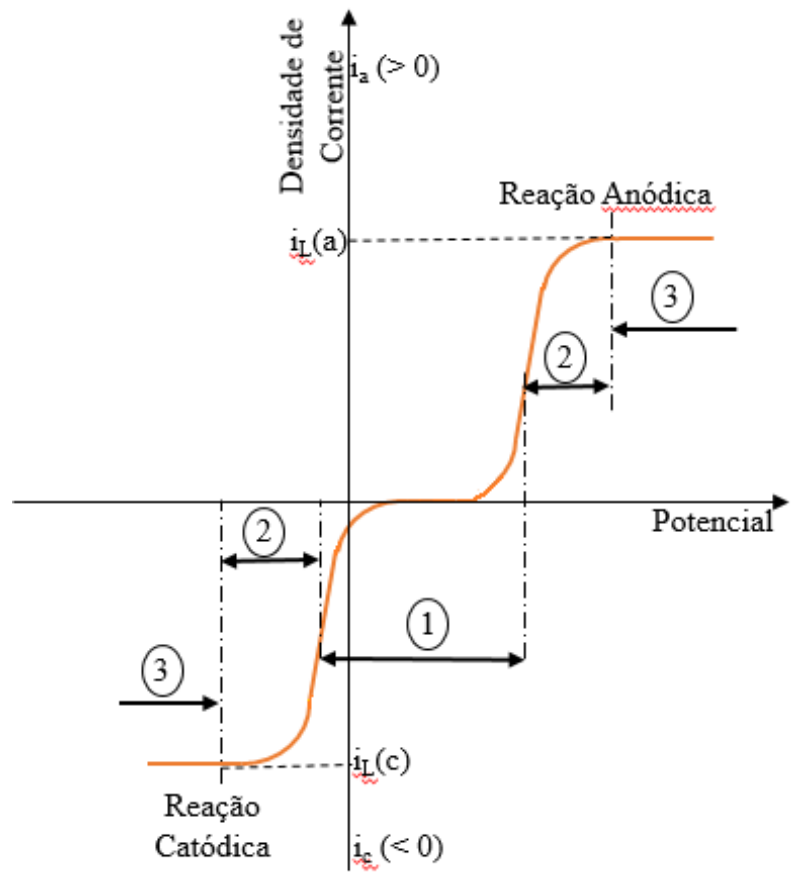

Segundo Ateya e Al-Kharafi (2002), após oxidação anódica o íon bissulfeto pode resultar em enxofre elementar $\left(S^{0}\right)$, polissulfetos $\left(S_{2}{ }^{2-}\right.$ e $\left.S_{3}{ }^{2-}\right)$, oxiânions de enxofre (sulfato $\left(\mathrm{SO}_{4}{ }^{2-}\right)$ e tiossulfato $\left.\left(\mathrm{S}_{2} \mathrm{O}_{3}{ }^{2-}\right)\right)$ com as seguintes reações e seus potenciais padrão $\left(\mathrm{E}^{0}\right)$ medidos em relação ao eletrodo padrão de hidrogênio (EPH):

$$
\begin{aligned}
& \mathrm{HS}^{-} € \mathrm{~S}^{0}+\mathrm{H}^{+}+2 \overline{\mathrm{e}} ; \mathrm{E}^{0}=-0,065 \mathrm{~V} \\
& 2 \mathrm{HS}^{-} € \mathrm{~S}_{2}^{2-}+2 \mathrm{H}^{+}+2 \overline{\mathrm{e}} ; \mathrm{E}^{0}=0,298 \mathrm{~V} \\
& 3 \mathrm{HS}^{-} € \mathrm{~S}_{3}^{2-}+3 \mathrm{H}^{+}+4 \overline{\mathrm{e}} ; \mathrm{E}^{0}=0,097 \mathrm{~V} \\
& \mathrm{HS}^{-}+4 \mathrm{H}_{2} \mathrm{O} € \mathrm{SO}_{4}^{2-}+9 \mathrm{H}^{+}+8 \overline{\mathrm{e}} ; \mathrm{E}^{0}=0,252 \mathrm{~V} \\
& 2 \mathrm{HS}^{-} € \mathrm{~S}_{2}^{2-}+2 \mathrm{H}^{+}+2 \overline{\mathrm{e}} ; \mathrm{E}^{0}=0,298 \mathrm{~V} \\
& 2 \mathrm{~S}^{0}+3 \mathrm{H}_{2} \mathrm{O} € \mathrm{~S}_{2} \mathrm{O}_{3}^{2-}+6 \mathrm{H}^{+}+4 \overline{\mathrm{e}} ; \mathrm{E}^{0}=0,165 \mathrm{~V}
\end{aligned}
$$


As reações (01) a (06) podem ocorrer, em princípio, simultaneamente se os íons $\mathrm{HS}^{-}\left(1 \mathrm{~mol} \cdot \mathrm{L}^{-1}\right)$ são oxidados num potencial padrão de $\mathrm{E}^{0}=0,35 \mathrm{~V}(\mathrm{EPH})$ (ATEYA e AL-KHARAFI, 2002).

Segundo Sergienko et al. (2019), os picos próximos a $0,3 \mathrm{~V}$ (vs. ECS) representam a oxidação de sulfeto em enxofre elementar atribuído à reação (01). Alguns picos subsequentes presentes em alguns voltamogramas, como na Fig 3(A), Fig 3(C) e Fig 4(A) representam a oxidação de sulfeto em oxiânios.

Após realização de cada experimento (total de 27) foi realizada a análise química da amostra para detectar a presença de $\mathrm{SO}_{4}{ }^{2-}$ e $\mathrm{S}_{2} \mathrm{O}_{3}{ }^{2-}$. Os resultados obtidos demonstraram a presença dos íons, confirmando o mecanismo apresentado por Ateya e Al-Kharafi (2002).

A Fig. 3 mostra os voltamogramas de varredura linear em função das concentrações de HS e $\mathrm{NaCl} 0,1 \mathrm{~mol} \cdot \mathrm{L}^{-1}$, a $298 \mathrm{~K}$. O pico de corrente anódica, também denominada corrente limite (i $\mathrm{i}_{\mathrm{L}}$ ) para a oxidação de íons sulfeto ocorreu a um potencial de aproximadamente $0,3(\mathrm{~V}$ vs. ECS) para as concentrações de $30 \mathrm{mg} \cdot \mathrm{L}^{-1}$ e $300 \mathrm{mg} \cdot \mathrm{L}^{-1}$. Isso demonstra que a superfície catalítica do eletrodo de trabalho de CVR para a oxidação de HS- encontra-se nesta faixa de potencial citada, nas condições experimentais aplicadas (KHUDAISH e AL-HINAI, 2006), indicando a ocorrência simultânea das reações indicadas pelas equações (01) a (06) (MATTIUSI, 2012).

Para a concentração de $3000 \mathrm{mg} \cdot \mathrm{L}^{-1}$ houve alteração na faixa de potencial em relação aos picos com a variação da velocidade de varredura aplicada, sendo que na velocidade de varredura de $10 \mathrm{mV} \cdot \mathrm{s}^{-1}$, o valor do potencial foi de aproximadamente 0,3 (V vs. ECS). Nesta concentração observou-se a relação linear $\left(\mathrm{R}^{2}=0,9\right)$ da corrente de pico $\left(\mathrm{I}_{\mathrm{p}}\right)$ com a raiz quadrada da velocidade de varredura de potencial (v) $\left(I_{p} \propto v^{1 / 2}\right)$ (BRETT, 1996). Segundo Khudaish e
Al-Hinai (2006), esta relação sugere que o processo do eletrodo é controlado por transporte de massa convectivo dos íons HS- para a superfície do eletrodo.

$\mathrm{Na}$ convecção o transporte de massa pode ocorrer devido à um gradiente de velocidade provocado por forças mecânicas, ou seja, o movimento do eletrólito próximo ao eletrodo se dá por agitação mecânica ou magnética, caracterizando a convecção forçada. Já a convecção natural pode ocorrer por gradiente de densidade ou gradiente de temperatura próximo à superfície do eletrodo (PLETCHER e WALSH, 1990; SCOTT, 1995; BARD e FAULKNER, 2001).

Para todas as soluções de $\mathrm{HS}^{-}$analisadas observou-se o aumento da corrente de pico com o aumento da velocidade de varredura, sendo que os valores de potencial de pico $\left(\mathrm{E}_{\mathrm{p}}\right)$ ficaram próximos a 0,3 (V vs. ECS). Este comportamento ocorre devido ao aumento da concentração de $\mathrm{HS}^{-}$na solução indicando o controle do sistema por transporte de massa. Os mesmos valores de $E_{p}$ indicam a ocorrência das mesmas reações em todos os voltamogramas expostos.

$\mathrm{O}$ aumento da corrente em potenciais acima de 0,9 (V vs. ECS) são atribuídos ao efeito da eletrólise da água e evolução de oxigênio na superfície do eletrodo (KHUDAISH e AL-HINAI, 2006).

$\mathrm{Na}$ Fig. 4 foram demonstrados os voltamogramas de varredura anódica das concentrações de $\mathrm{HS}^{-}$e $\mathrm{NaCl}$ 0,1 mol $\cdot \mathrm{L}^{-1}$, a $298 \mathrm{~K}$, em diferentes rotações do eletrodo de trabalho para a velocidade de varredura de potencial de $1 \mathrm{mV} \cdot \mathrm{s}^{-1}$. Nesta velocidade observou-se uma diferença entre os perfis em relação às outras velocidades analisadas (Fig. 3). Este comportamento pode ser atribuído ao regime estacionário estabelecido em tais condições. $\mathrm{O}$ aumento da corrente de pico com o aumento da rotação indica que não há passivação do eletrodo por deposição de enxofre elementar (KHUDAISH e ALHINAI, 2006). 
Figura 3. Voltamogramas de solução sintética, corrente (uA) em função do potencial (V vs. ECS) em diferentes velocidades de varredura: (A) $30 \mathrm{mg} \cdot \mathrm{L}^{-1}$, (B) $300 \mathrm{mg} \cdot \mathrm{L}^{-1}$, (C) $3000 \mathrm{mg} \cdot \mathrm{L}^{-1}$.



(A)

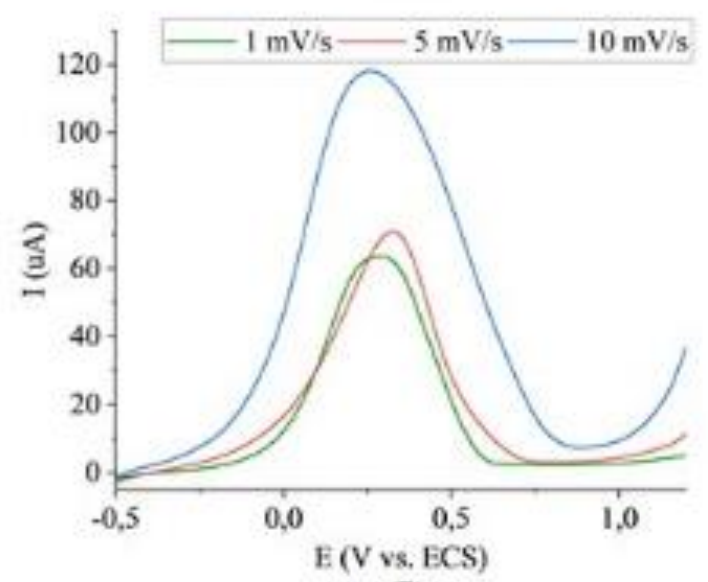

(B)

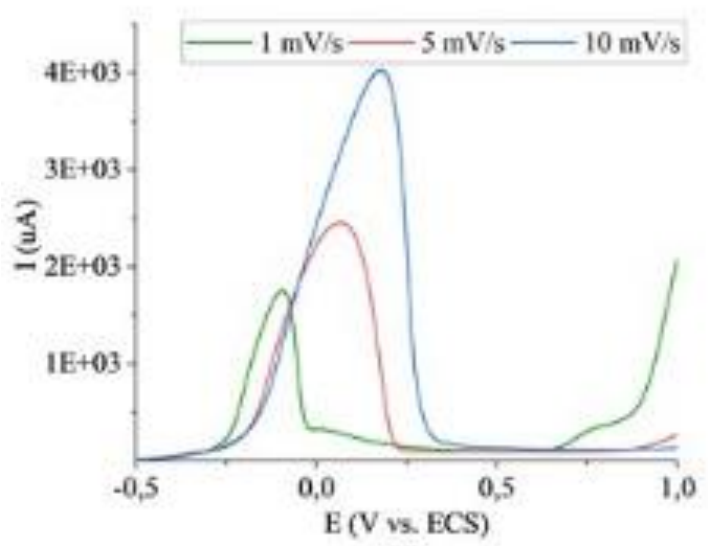

(C)

Nos voltamogramas demonstrados na Fig. 4 é possível observar que, da mesma forma que nos voltamogramas da Fig. 3, o potencial de pico encontrase em aproximadamente 0,3 (V vs. ECS) para as rotações de $50 \mathrm{rpm}$ e $100 \mathrm{rpm}$. A corrente de pico para alta concentração de $\mathrm{HS}^{-}$foram elevadas independente da rotação aplicada. Essa tendência era esperada pois o fluxo na célula diminui a espessura da camada limite na superfície do eletrodo facilitando o transporte dos íons à superfície, e subsequentemente a ocorrência das reações de oxidação. Além disso, devido à rotação, o produto é rapidamente removido da vizinhança do eletrodo.

Figura 4. Voltamogramas de solução sintética, corrente (uA) em função do potencial (V vs. ECS) para velocidade de varredura de $1 \mathrm{mV} \cdot \mathrm{s}^{-1} \mathrm{em}$ diferentes rotações do eletrodo de trabalho: (A) $30 \mathrm{mg} \cdot \mathrm{L}^{-1}$, (B) $300 \mathrm{mg} \cdot \mathrm{L}^{-1}$, (C) $3000 \mathrm{mg} \cdot \mathrm{L}^{-1}$.

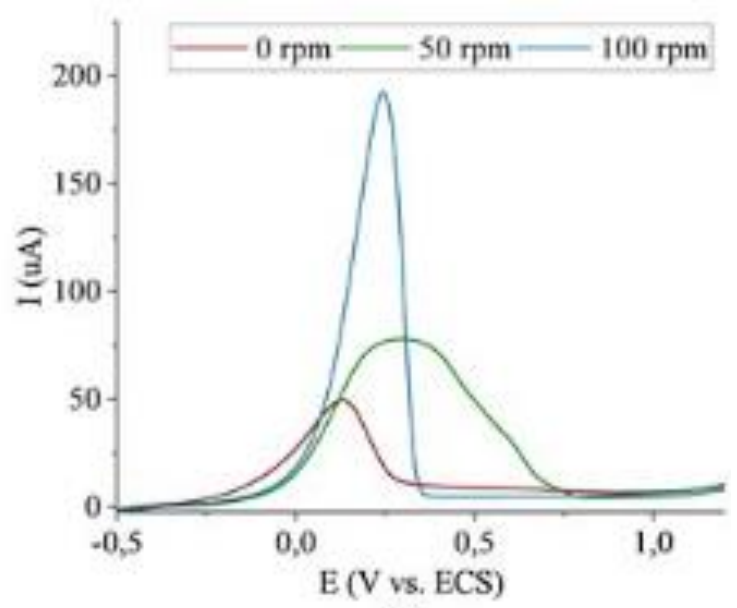

(A)

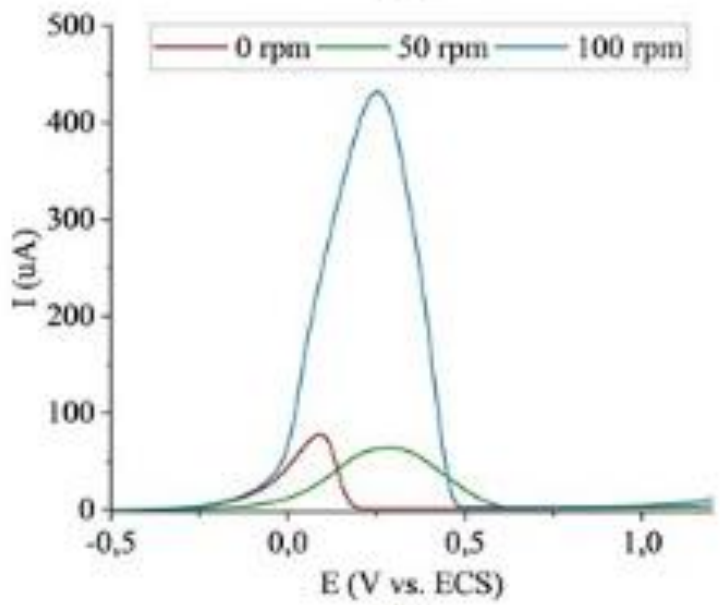

(B)

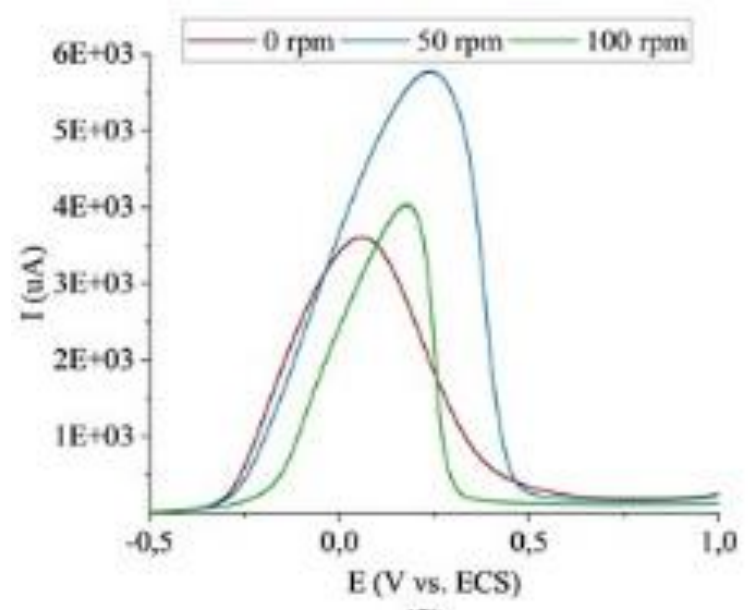

(C) 


\section{CONCLUSÃO}

O presente trabalho revela a possibilidade de oxidar eletroquimicamente íons sulfeto em soluções aquosas utilizando eletrodo de carbono vítreo reticulado. Os resultados obtidos indicaram que o potencial no qual ocorrem as reações de interesse fica próximo a $0,3 \mathrm{~V}$ (vs. ECS), o que sugere a presença das mesmas reações para todos os casos analisados. Nos voltamogramas foi possível observar um aumento proporcional da corrente de pico com a taxa de varredura e concentração, indicando o controle do sistema por transporte de massa. Os resultados demostraram que o enxofre elementar não passiva a superfície do eletrodo. Sendo assim, o enxofre convertido pode ser subsequentemente removido do efluente.

\section{AGRADECIMENTO}

Laboratório de Tecnologia Ambiental (LTA) por possibilitar a realização dos experimentos, à Coordenação de Aperfeiçoamento de Pessoal de Nível Superior (CAPES) e ao Programa de Recursos Humanos da Agência Nacional do Petróleo, Gás Natural e Biocombustíveis - PRH-ANP pelo apoio financeiro.

Todos os autores declararam não haver qualquer potencial conflito de interesses referente a este artigo.

\section{REFERÊNCIAS}

ATEYA, B.G.; AL-KHARAFI, F.M. Anodic Oxidation of Sulfide Ions from Chloride Brines. Electrochemistry Communications. v. 4, p. 231-238, 2002.

ATEYA, B. G.; AL-KHARAFI, F.M.; ABDALLAH, R. M.; Al-AZAB, A.S. Electrochemical Removal of Hydrogen Sulfide from Polluted Brines Using Porous Flow Through Electrodes. Journal of Applied Electrochemistry. v. 35, p. 297-303, 2005.

BARD, A.J.; FAULKNER, L.R. Electrochemical

Methods: Fundamentals and Applications. 2 ed. New York: John Wiley \& Sons, 2001.
BERNÉ, F., CORDONNIER, J. Industrial Water Treatment - Refining Petrochemicals and Gas Processing Techniques. Texas: Gulf Professional Publishing, 1995.

BRETT, A. M.; BRETT, C. M. A. Electroquímica: Princípios, Métodos e Aplicações. Livraria Almedina, Coimbra, 1996.

COEURET, F. Introducción a la Ingeniería Electroquímica. Espanha: Reverté, 1992.

DUTTA, P. K.; RABAEY, K.; YUAN, Z.; KELLER, J. Spontaneous Electrochemical Removal of Aqueous Sulfide. Water Research, v. 42, n. 20, p. 4965-4975, 2008.

DUTTA, P. K.; ROZENDAL, R. A.; YUAN, Z.; RABAEY K.; KELLER, J. Electrochemical Regeneration of Sulfur Loaded Electrodes. Electrochemistry Communications, v. 11, n. 7, p. 1437-1440, 2009.

DUTTA, P.K.; RABAEY, K.; YUAN, Z.; ROSENDAL, R. A.; KELLER, J. Electrochemical Sulfide Removal and Recovery from Paper Mill Anaerobic Treatment Effuent. Water Research. v. 44, n. 8, p. 2563-2571, 2010.

FORNÉS, J. P.; BISANG, J. M. Electrochemical Production of Colloidal Sulphur by Oxidation of Sulphide Ion at Lead Coated-2-and-3-Dimensional Rotating Cylinder Anode Surfaces. Electrochimica Acta. v. 243, p. 90-97, 2017.

JÜTTNER, K.; GALLA, U.; SCHMIEDER, H. Electrochemical Approaches to Environmental Problems in the Process Industry. Electrochimica Acta. v. 45, n. 15-16, p. 2575-2594, 2000.

KHUDAISH, E. A.; AL-HINAI, A. T. The Catalytic Activity of Vanadium Pentoxide Film Modified Electrode on the Electrochemical Oxidation of Hydrogen Sulfide in Alkaline Solutions. Journal of Electroanalyctical Chemistry. v. 587, n. 1, p. 108114, 2006.

LAM, V.; LI, G.; SONG, C.; CHEN, J.; FAIRBRIDGE, C.; HUI, R.; ZHANG, J. A Review of Electrochemical Desulfurization Technologies for Fossil Fuels. Fuel Processing Technology. v. 98, p. 30-38, 2012.

MAINIER, F. B.; SANDRES, G. C.; TAVARES, S. S. M. Corrosão por Sulfeto de Hidrogênio e suas Implicações no Meio Ambiente e na Segurança Industrial. $8^{\circ}$ Congresso Ibero Americano de Engenharia Mecânica. Cusco, 2007. 
MATTIUSI, E. M. Análise do Comportamento de um Reator Eletroquímico de Leito Poroso no Tratamento de Efluentes Industriais Contaminados por Sulfeto de Hidrogênio. 2012. Tese (Doutorado) - Universidade Federal do Paraná, Programa de Pós-Graduação em Engenharia Mecânica - PPGEM. Defesa: Curitiba, 2012.

MATTIUSI, E.M.; KAMINARI, N.M.S.; PONTE, M.J.J.S.; PONTE, H.A. Behavior Analysis of a Porous Bed Electrochemical Reactor the Treatment of Petrochemical Industry Wastewater Contaminated by Hydrogen Sulfide $\left(\mathrm{H}_{2} \mathrm{~S}\right)$. Chemical Engineering Journal. v. 275, p. 305-314, 2015.

NTAGIA, E.; FISET, E.; LIMA, L. S.; PIKAAR, I.; ZHANG, X.; JEREMIASSE, A. W.; PRÉVOTEAU, A.; RABAEY, K. Anode Materials for Sulfide Oxidation in Alkaline Wastewater: An Activity and Stability Performance Comparison. Water Research. v. 149, p. 111-119, 2019.

PERCHERON, G.; BERNET, N.; MOLETTA, R. A New Method for the Determination of Dissolved Sulfide in Strongly Colored Anaerobically Treated Effluents. Bioprocess Engineering. v. 15, p. 317-322, 1996.

PERRY, S. C.; PONCE DE LEÓN, C.; WALSH, F. C. Review - The Design, Performance and Continuing Development of Electrochemical Reactors for Clean Electrosynthesis. Journal of the Electrochemical Society. v. 167, 155525, 2020.

PIKAAR, I.; ROZENDAL, R. A.; YUAN, Z.; KELLER, J.; RABAEY, K. Electrochemical Sulfide Removal from Synthetic and Real Domestic
Wastewater at High Current Densities. Water Research. v. 45, p. 2281-2289, 2011.

PIKAAR, I.; LIKOSOVA, E. M.; FREGUIA, S.; KELLER, J.; RABAEY, K.; YUAN, Z. Electrochemical Abatement of Hydrogen Sulfide from Waste Streams. Critical Reviews Environmental Science and Technology. v. 45, p. 1555-1578, 2015.

PLETCHER, D; WALSH, F. C. Industrial Electrochemistry. 2 ed. Londres: Chapman and Hall, 1990.

SCOTT, K. Reaction Engineering and Digital Simulation in Electrochemical Processes. In: WHITE, R. E.; BOCKRIS, J. O'M.; CONWAY, B. E. Modern Aspects of Electrochemistry. Springer Science, v. 27, p. 1-142, N. Y., 1995.

SERGIENKO, N.; IRTEM, E.; GUTIERREZ, O.; RADJENOVIC, J. Electrochemical Removal of Sulfide on Porous Carbon Based Flow-Through Electrodes. Journal of Hazardous Materials, v. 375, p. 19-25, 2019.

VAIOPOULOU, E.; PROVIJIN, T.; PRÉVOTEAU, A.; PIKAAR, I.; RABAEY, K. Electrochemical Sulfide Removal and Caustic Recovery from Spent Caustic Streams. Water Research, v. 92, p. 38-43, 2016.

WALSH, F. C.; ARENAS, L. F.; PONCE DE LEÓN, C.; READE, G. W.; WHYTE, I.; MELLOR, B. G. The continued development of reticulated vitreous carbon as a versatile electrode material: Structure, properties and applications. Electrochimica Acta. v. 215, p. 566591, 2016. 